\title{
Cerebellar Cortex Lesions Prevent Acquisition of Conditioned Eyelid Responses
}

\author{
Keith S. Garcia, Philip M. Steele, and Michael D. Mauk \\ Department of Neurobiology and Anatomy and The Keck Center for the Neurobiology of Learning and Memory, University \\ of Texas Medical School, Houston, Texas 77030
}

\begin{abstract}
We have used aspiration and electrolytic lesions to investigate the contributions of cerebellar cortex to the acquisition and expression of conditioned eyelid responses. We show that lesions of the anterior lobe of rabbit cerebellar cortex disrupt the timing of previously learned conditioned eyelid responses. These short-latency responses were used as an indication that the cerebellar cortex was sufficiently damaged and that the underlying pathways necessary for the expression of responses were sufficiently intact to support responses. Rabbits were subsequently trained for 15 daily sessions using a new conditioned stimulus. Whereas rabbits in which lesions had no significant effect on response timing showed rapid acquisition of appropriately timed eyelid responses to the new conditioned stimulus, animals with lesions that disrupt timing showed no
\end{abstract}

significant increases in either amplitude or probability of responses. Histological analysis suggests that damage to the anterior lobe of the cerebellar cortex is necessary and sufficient to abolish timing and prevent acquisition. These data indicate that the cerebellar cortex is necessary for the acquisition of conditioned eyelid responses and are consistent with the hypotheses that (1) eyelid conditioning results in plasticity in both the anterior lobe of the cerebellar cortex and in the anterior interpositus nucleus and (2) induction of plasticity in the interpositus requires intact input from the cerebellar cortex.

Key words: Pavlovian eyelid conditioning; classical eyelid conditioning; nictitating membrane; LTP; LTD; motor learning; extinction; timing; deep cerebellar nucleus
Since the publication of Marr's (1969) seminal theory, the role of the cerebellar cortex in motor learning has been intensely debated. Although evidence indicates that Pavlovian eyelid conditioning is one of several examples of motor learning mediated by the cerebellum (Ritchie, 1976; Robinson, 1976; Gilbert and Thach, 1977; Thach, 1980; Thompson, 1986; Lisberger, 1988; Robinson et al., 1993; Thompson and Krupa, 1994), the relative contributions of cerebellar cortex and cerebellar nuclei are a source of controversy (McCormick and Thompson, 1984a; Yeo et al., 1985a; Lisberger and Sejnowski, 1992; Ito, 1993; Mauk, 1997). Here we address the necessity of the cerebellar cortex for the acquisition of conditioned eyelid responses. Previous studies indicate that presentation of the conditioned stimulus (CS) is conveyed to the cerebellum via mossy fiber afferents (Steinmetz et al., 1986, 1987, 1989), the reinforcing properties of the unconditioned stimulus (US) require activation of climbing fiber afferents (McCormick et al., 1985; Mauk et al., 1986; Yeo et al., 1986), and activation of the appropriate neurons in the anterior interpositus nucleus drives the expression of conditioned responses (McCormick and Thompson, 1984a,b; Chapman et al., 1988). There are two cerebellar pathways by which CS-activated mossy fibers could come to activate interpositus neurons during training-the excitatory mossy fiber synapses directly onto the output neurons and the more complex pathway through the cerebellar cortex (Fig. 1A). Evidence suggests that both pathways can undergo plasticity during eyelid conditioning (McCormick and

\footnotetext{
Received June 15, 1999; revised Sept. 16, 1999; accepted Sept. 24, 1999.

This work was supported by Grants MH 46904 and MH 57051.

Correspondence should be addressed to Dr. Michael D. Mauk, Department of Neurobiology and Anatomy, University of Texas Medical School, P.O. Box 20708, Houston, TX 77225. E-mail: mmauk@nba19.med.uth.tmc.edu.

Copyright (C) 1999 Society for Neuroscience 0270-6474/99/1910940-08\$05.00/0
}

Thompson, 1984a; Krupa et al., 1993; Perrett et al., 1993; Hesslow and Ivarsson, 1994; Tracy et al., 1998) and during adaptation of the vestibulo-ocular reflex (VOR) (Miles and Lisberger, 1981; Ito, 1993; Lisberger and Raymond, 1996; Raymond et al., 1996). In essence, our experiments ask whether the direct pathway through the cerebellar nucleus can support eyelid conditioning without the cerebellar cortex (Fig. 1B).

Interpreting the effects of cerebellar cortex lesions on the acquisition of conditioned eyelid responses requires assurance that a lesion has damaged appropriate regions of cortex but has spared underlying pathways necessary for response expression. For example, in previous studies reporting normal or only partially compromised acquisition, it is not clear that relevant regions of the cerebellar cortex were damaged (McCormick and Thompson, 1984a; Lavond et al., 1987; Lavond and Steinmetz, 1989; Yeo and Hardiman, 1992; Harvey et al., 1993; Clark and Lavond, 1994; Gruart and Yeo, 1995). Similarly, in studies showing abolished eyelid response acquisition, the functional integrity of the interpositus nucleus was unclear (Yeo et al., 1984, 1985a). Here, we attempt to avoid these shortcomings by using the effects of cerebellar cortex removal on the expression of conditioned responses as an assay for the effectiveness and specificity of the lesions. We have shown previously that either blockade of cerebellar cortex output (Garcia and Mauk, 1998b) or lesions of the cerebellar cortex (Perrett et al., 1993; Perrett and Mauk, 1995) abolish the timing of eyelid responses, yielding responses with short and relatively fixed latencies to onset. Because evidence indicates that the short-latency responses are learned and are abolished by lesions of the interpositus nucleus (Perrett and Mauk, 1995; Garcia and Mauk, 1998a,b), the short-latency responses can be used as a functional readout that a lesion has 

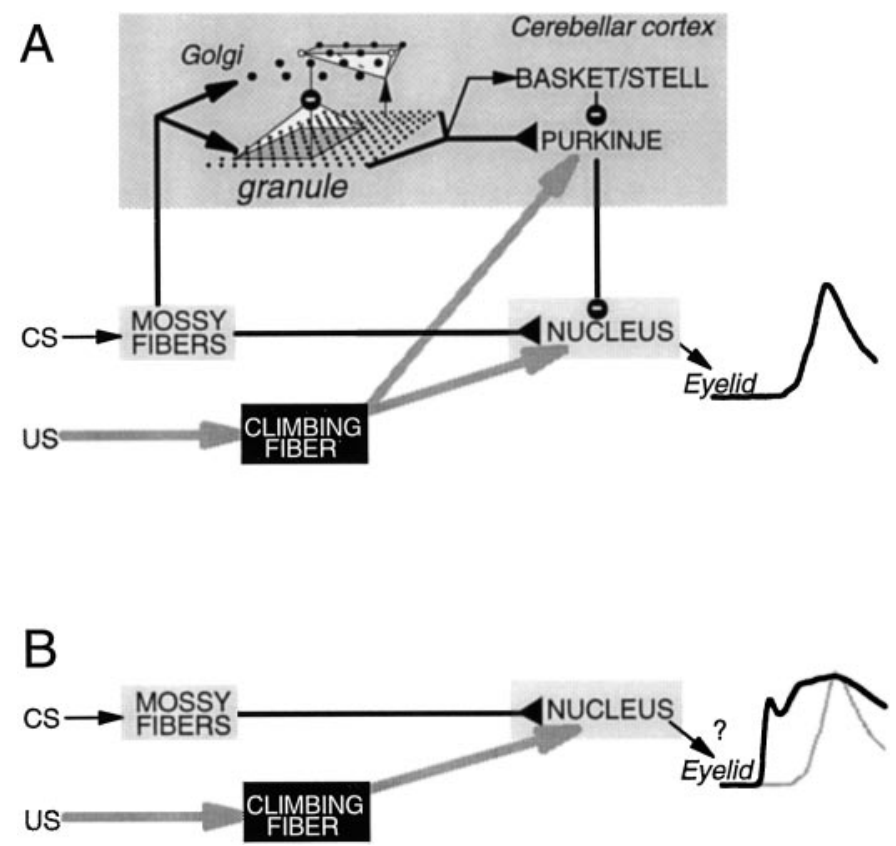

Figure 1. A, A schematic representation of the basic features of cerebellar connectivity and its relationship to Pavlovian eyelid conditioning. The CS is conveyed to the cerebellum via activation of particular mossy fibers, and the US activates particular climbing fibers. Activity in particular neurons in the cerebellar anterior interpositus nucleus (NUCLEUS) is responsible for the expression of the conditioned eyelid responses. $B, \mathrm{~A}$ schematic representation of the question addressed in the present experiments: is acquisition possible without the cerebellar cortex?

damaged critical regions of cerebellar cortex without damaging the interpositus nucleus or other pathways necessary for response expression.

We show that when cerebellar cortex lesions meet these criteria, animals are subsequently unable to acquire conditioned eyelid responses using a new conditioned stimulus. The ability of the previously trained CS to elicit responses excludes the possibility that the inability to acquire responses is really a deficit in response expression. In concert with previous work, these findings support the hypothesis that eyelid conditioning involves plasticity in both the cerebellar cortex and nuclei and that the cerebellar cortex is required both for acquisition and extinction of responses as well as for the induction of plasticity in the interpositus nucleus.

\section{MATERIALS AND METHODS}

Animals. Data are presented from 14 male New Zealand albino rabbits (Oryctolagus cuniculus), weighing $2.5-3.0 \mathrm{~kg}$ each at the start of the experiments. The animals were individually housed and fed food and water ad libitum. Treatment of the animals and surgical procedures were in accordance with an approved animal welfare protocol.

Surgery. All animals were first surgically prepared with a head bolt cemented to the skull. Of these, 2 animals were also prepared with electrodes implanted in the anterior lobe of the cerebellar cortex, and the remaining 12 animals later received aspiration lesions after initial training.

Surgical procedures for aspiration lesions were identical to those described previously (Perrett and Mauk, 1995). For the electrolytic lesion animals, the following procedures were used. A large craniotomy was drilled to accommodate the electrode assembly or a cannula and covered with bone wax. The electrode assembly consisted of an array of four stainless steel electrodes equally spaced in a linear strip $\sim 3 \mathrm{~mm}$ in length. The distal $1 \mathrm{~mm}$ of each electrode was exposed by scraping off the insulation with a scalpel blade. The head of the rabbit was positioned with lambda $1.5 \mathrm{~mm}$ ventral to bregma. The array was placed in a parasagittal orientation. The anteriormost electrode was placed at stereotaxic coordinates corresponding to the lateral anterior lobe $(5.7 \mathrm{~mm}$ anterior, $4.9 \mathrm{~mm}$ left lateral, and $14.0 \mathrm{~mm}$ ventral to lambda). After placement, the electrode assembly and head bolt were secured to the skull with dental acrylic, and the skin was sutured. Two stainless steel stimulating electrodes were chronically implanted in the periorbital muscles rostral and caudal to the eye. Antibiotics, intravenous fluids, and analgesics were administered after surgery as needed, and animals were allowed $\sim 1$ week to recover.

Conditioning procedures. The standard training session involved a Pavlovian conditioning delay protocol with a $500 \mathrm{msec}$ interstimulus interval. Each training session consisted of 12 nine-trial blocks. Each block was comprised of eight paired presentations of the CS and US and one presentation of the CS only. The CS (a $1 \mathrm{kHz}, 85 \mathrm{~dB}$ tone or a vibrator secured to the animal's back) was presented for $550 \mathrm{msec}$ during CSalone trials and coterminated with a $50 \mathrm{msec}$ train of constant-current pulses $(200 \mathrm{~Hz} ; 1 \mathrm{msec}$ pulse width; $2-3 \mathrm{~mA})$ delivered to the periorbital electrodes during paired trials. The intensity of the US was adjusted individually for each animal to produce a robust unconditioned response without overt signs of discomfort. Trials were separated by a fixed $30 \mathrm{sec}$ intertrial interval.

Animals were conditioned to an asymptotic rate of responding with at least 10 standard training sessions. Lesions were then made either by passing $2.0 \mathrm{~mA}$ of direct current through one or more of the electrodes for $\sim 1 \mathrm{~min}$ or by surgical aspiration as described previously.

In these animals the effect of lesions on timing was assessed with two postlesion training sessions. Animals with lesions then received $15 \mathrm{~d}$ of postlesion acquisition training, each consisting of 1210 -trial blocks. Each block was of eight paired presentations of the novel CS with the US and one presentation each of both the pre- and postlesion CS alone. The tone and vibrator were counterbalanced with regard to pre- and postlesion training. Finally, animals that failed to acquire responses after the lesion received $2 \mathrm{~d}$ of training the contralateral eyelid to the postlesion CS.

Stimulus presentation and data acquisition were computer-controlled using custom software. Movement of the unrestrained eyelid was recorded by measuring the reflectance of an infrared light-emitting diode aimed at the eyelid. Voltage responses were determined to be linearly related to eyelid movement and were calibrated for each animal daily. Digitized responses (1 point per msec; 12 bit resolution) were analyzed using custom software to determine onset and peak latencies.

Histology. After training, the location of the lesion was determined for each animal using standard histological procedures. Animals were killed with an overdose of sodium pentobarbital and perfused intracardially with 1.01 of $10 \%$ formalin. The brains were removed and stored in $10 \%$ formalin for several days. Brains were embedded in an albumin gelatin mixture. To assess the extent of aspiration or electrolytic lesions, we sectioned the cerebellum parasagittally using a freezing microtome ( 80 $\mu \mathrm{m}$ sections). This plane of sectioning best illustrated the degree to which tissue damage involved each lobule of cerebellar cortex.

Data analysis and statistical tests. Peak response amplitude and onset latency were calculated by custom software. Digitized sweeps corresponded to the $200 \mathrm{msec}$ before and $2300 \mathrm{msec}$ after the CS onset. After calibration, peak amplitude was measured relative to an average of the $200 \mathrm{msec}$ baseline collected before CS onset. Onset latency was determined by calculating the point at which the response slope deviated by two SDs from the average slope of the baseline. To be counted as a conditioned response, onset latency had to follow CS onset, and the amplitude was required to reach $0.3 \mathrm{~mm}$ before US onset during paired trials. This criterion was relaxed for CS-alone trials in which movements were counted as conditioned responses if they reached a $0.3 \mathrm{~mm}$ amplitude at any time after CS onset. Trials in which there was $>0.3 \mathrm{~mm}$ of movement during the baseline were excluded.

Statistical analysis included tests for the effects of the lesions on response timing for each individual animal and tests for group effects of the lesions on acquisition using amplitude and percent response as dependent measures. Animals were separated into "lesion" or "control" groups on the bases of separate one-tailed $t$ tests performed on peak and onset latencies from the last 12 conditioned responses before the lesion and the first 12 conditioned responses after the lesion $(p<0.01$ criterion). A two-way, mixed ANOVA was performed on the postlesion response rate to compare groups with regard to acquisition with the novel CS. Response rate data from the lesion group during the 15 postlesion training days were further assessed using a one-way, repeated measures 
ANOVA. A minimum confidence level of $p<0.05$ was used for these group analyses.

\section{RESULTS}

The ability to evaluate the effects of the lesions on a previously trained response is the key feature of the experimental design. Before aspiration or electrolytic lesions of the cerebellar cortex, animals were trained to make robust eyelid responses to either a tone or a tactile vibratory CS applied to the back. After lesions, animals were grouped according to the effects of the lesions on response timing. Animals were assigned to the experimental group only if lesions decreased both peak and onset latencies. Five animals reported in this study demonstrated significant effects $(p<0.001)$ on both onset and peak latency as determined by separate $t$ tests. Cortex lesions had similar effects on response timing whether the original CS was a $1 \mathrm{kHz}$ tone or the vibratory stimulus. Several animals with cerebellar cortex lesions demonstrated clear effects on the latency to onset but not the latency to response peak. This pattern of results, which appears to result from partial lesions of the anterior lobe, will be reported in a separate study.

To assess postlesion acquisition, animals were then trained to a new CS. Those that were initially trained to the tone received $15 \mathrm{~d}$ of training using the vibratory $\mathrm{CS}$, and animals trained to the vibrator before the lesion were trained using the tone CS. We find that animals in which cortex lesions disrupted response timing were unable to acquire either short-latency or timed responses when trained with the new CS, although presentation of the prelesion CS continued to elicit robust short-latency responses (Fig. 2). The animals sometimes displayed small, short-latency responses to the new CS, presumably because of the slight auditory component of the vibratory stimulus. Although these animals show no increases in responding to the new CS, they rapidly acquired well timed responses when the eye contralateral to the lesion was trained. In contrast, when response timing was not affected by the cerebellar cortex lesion, animals rapidly acquired well timed conditioned responses to the novel CS.

The CS-alone trials (original CS) intermixed during the $15 \mathrm{~d}$ of postlesion training also permitted evaluation of extinction in the control and lesion animals (Fig. 3). Although extinction in the control animals was modest, presumably owing to the small number of trials presented, the decline in responding was significant. In contrast, in both the amplitude and percent response measures the lesion animals showed no apparent decreases in short-latency responding to the original CS.

Statistical analyses of both amplitude and percent-responding data indicate that the lesions prevented acquisition of responses to the new CS (i.e., Fig. 2). Separate two-way, mixed ANOVAs were performed for both amplitude and percent-responding data. For the new CS data there were main effects of lesion versus control [percent data $F_{(1,12)}=118.69 ; p<0.001$; amplitude data $\left.F_{(1,12)}=233.87 ; p<0.001\right]$. There were also significant main effects for days [percent data $F_{(14,168)}=7.85 ; p<0.001$; amplitude data $\left.F_{(14,168)}=3.12 ; p<0.025\right]$. The interaction effect of lesion by day was significant only for the percent-responding data [percent data $F_{(14,168)}=4.50 ; p<0.005$; amplitude data $F_{(14,168)}$ $=1.96 ; \mathrm{NS}]$. As an additional post hoc test, one-way repeated measures ANOVAs were calculated separately for data from lesion animals and from control animals. These analyses show no significant change in responding in the lesion group over the $15 \mathrm{~d}$ of postlesion training [percent data $F_{(14,56)}=1.69 ;$ NS; amplitude data $\left.F_{(14,56)}=1.62 ; \mathrm{NS}\right]$. In contrast, similar analysis of the
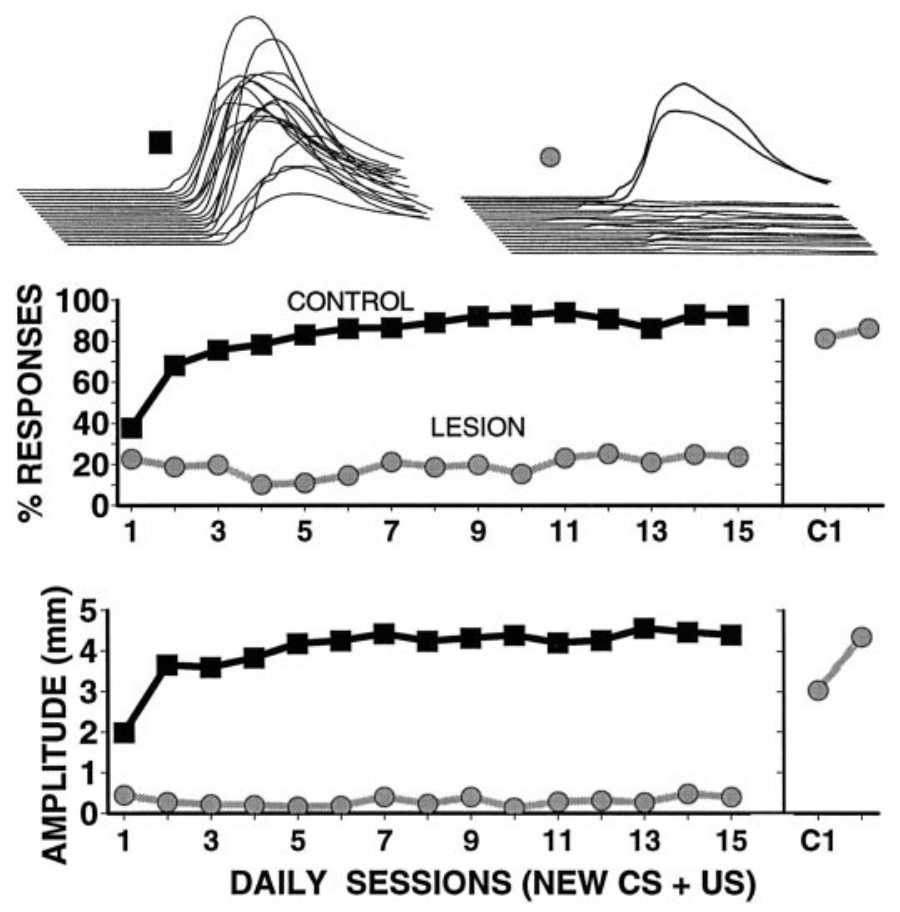

Figure 2. Middle, Bottom, Group data showing responding to the new CS (percent responses, middle; amplitude of responses, bottom) that shows that lesions of the cerebellar cortex prevent acquisition of conditioned eyelid responses. Whereas the control animals (dark squares; $n=9$ ) acquire robust conditioned eyelid responses over the $15 \mathrm{~d}$ of postlesion training, the lesion animals ( gray circles; $n=5$ ) show no apparent increase in responding. In the lesion animals, training was switched to the contralateral eye after $15 \mathrm{~d}(\mathrm{Cl}$; right) showing that the effects of the lesions are specific to the ipsilateral eye. Top, Sample responses from one control (left) and one lesion (right) animal. Each sweep is an average of the $\mathrm{CS}$-alone test trials for a session. The large responses for the lesion animal are from the contralateral eye.

control data showed significant acquisition over $15 \mathrm{~d}$ of training [percent data $F_{(14,112)}=8.79 ; p<0.001$; amplitude data $F_{(14,112)}$ $=3.38 ; p<0.01]$.

Identical analyses of the original CS data indicate that the lesions of cerebellar cortex prevented extinction of responding to the CS trained before the lesion (i.e., Fig. 3). Separate two-way, mixed ANOVAs (percent and amplitude data) revealed a main effect of the lesion only for the amplitude data [percent data $F_{(1,12)}=0.04 ;$ NS; amplitude data $\left.F_{(1,12)}=21.61 ; p<0.001\right]$. This indicates that the lesions had a significant effect on the amplitude of previously trained responses but not on their likelihood. There were main effects for days [percent data $F_{(14,168)}=4.03 ; p<$ 0.005 ; amplitude data $\left.F_{(14,168)}=4.19 ; p<0.005\right]$. Importantly, for both response measures there was a significant lesion-by-day interaction effect [percent data $F_{(14,168)}=2.73$; $p<0.025$; amplitude data $\left.F_{(14,168)}=2.56 ; p<0.025\right]$. As additional tests, one-way repeated measures ANOVAs were calculated separately for data from lesion and from control animals. For both response measures the results indicate a significant extinction in control animals [percent data $F_{(14,112)}=5.65 ; p<0.001$; amplitude data $\left.F_{(14,112)}=4.86 ; p<0.001\right]$. Similar analyses for the lesion animal data showed that there was no significant change in responding to the original CS over the 15 postlesion days [percent data $F_{(14,56)}$ $=0.60 ; \mathrm{NS}$; amplitude data $F_{(14,56)}=0.41$; NS].

Histological reconstruction of the lesions revealed a consistent relationship between damage to cerebellar lobules $\mathrm{HIV} / \mathrm{V}$ and 

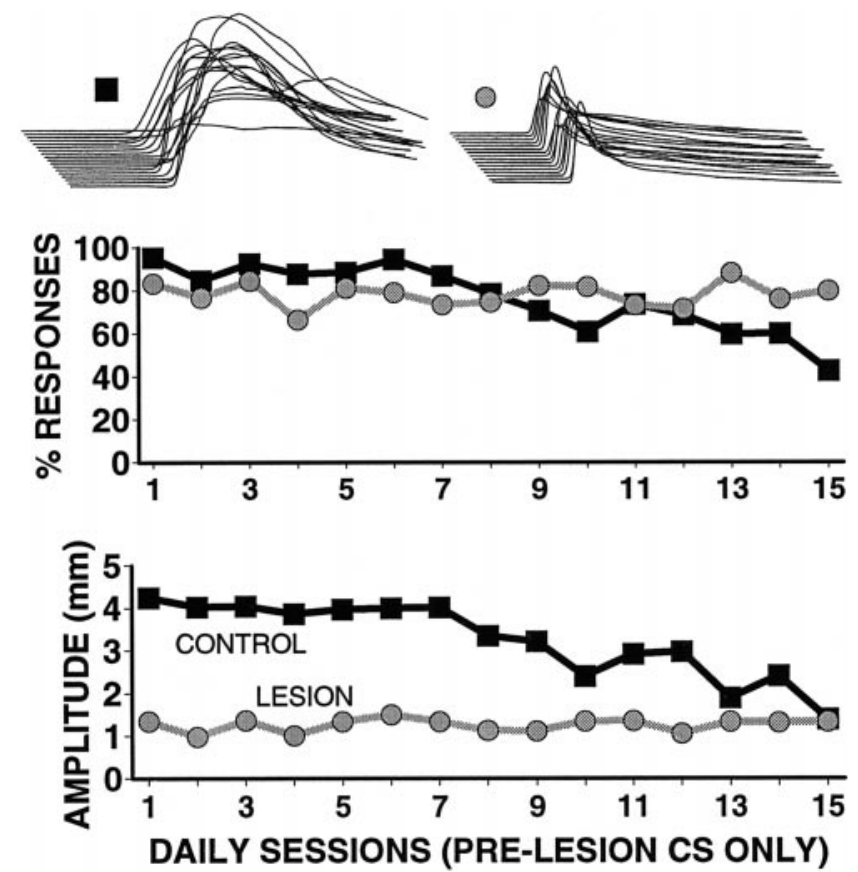

Figure 3. Group data showing responding to the original CS. Middle, Percent responding. Bottom, Response amplitude. Whereas the control animals (dark squares; $n=9$ ) showed a decrement in responding to the original CS, lesion animals (gray circles; $n=5$ ) did not. Top, Sample responses from one control (left) and one lesion (right) animal. The animals and formatting of the responses are the same as in Figure 2.

the effects observed on both timing and acquisition. Large lesions that included most of HVI but did not involve HIV/V neither affected timing nor prevented acquisition. Figure 4 depicts the smallest effective and the largest ineffective lesions. All of the effective lesions and none of the ineffective lesions included the lateral aspect of $\mathrm{HIV} / \mathrm{V}$. Damage to HVI had no effect on response timing or acquisition. One animal with a small electrolytic lesion illustrated especially clearly the role of the anterior lobe (Fig. 5). In this animal, visible damage was limited to the anterior lobe, with no histologically detectable damage to lobule HVI. This animal showed a robust effect on response timing and a complete inability to acquire responses to the new CS. Together these data demonstrate that the anterior lobe of the cerebellar cortex is required for the acquisition and expression of timed responses and plays a critical role in the training-induced plasticity underlying the acquisition of short-latency responses as well.

\section{DISCUSSION}

We have demonstrated that lesions of the cerebellar cortex abolish the learned timing of conditioned eyelid responses and prevent acquisition of responses to a new CS. Although extinction in control animals was modest because of the paucity of CS-alone trials, the present data also replicate previous findings that lesions of the cerebellar cortex prevent extinction of previously learned responses. In contrast, large lesions that do not affect the anterior lobe, including those with extensive damage of lobule HVI, neither affect response timing, prevent extinction, nor prevent acquisition. The data also show that these lesion effects are not restricted to auditory stimuli. These data demonstrate that the cerebellar cortex is necessary for the acquisition and extinction of conditioned eyelid responses and suggest that the cerebellar cor-

\section{SMALLEST EFFECTIVE LESION}

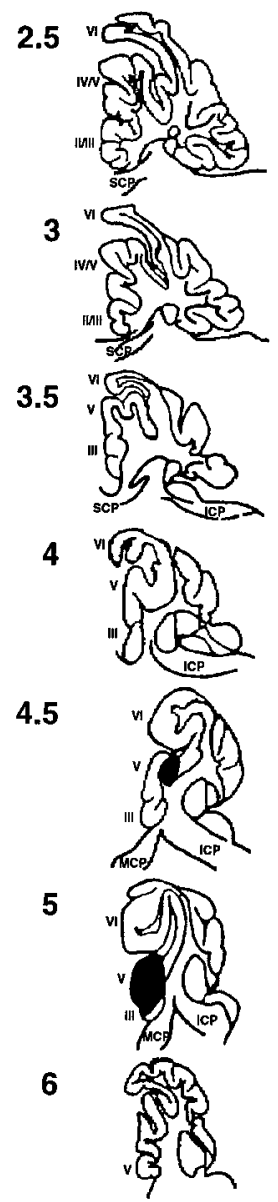

\section{LARGEST \\ CONTROL LESION}

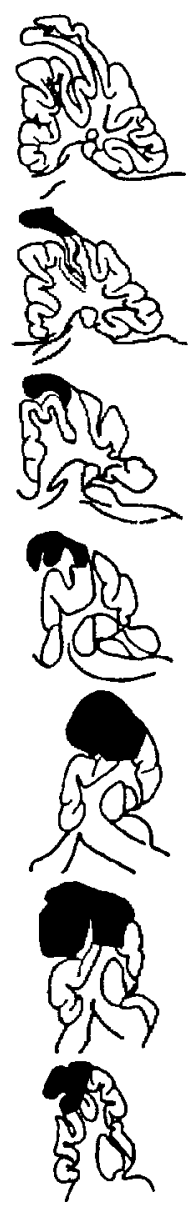

Figure 4. Schematic reconstructions, shown in parasagittal view, of the smallest effective lesion (left column) and the largest control lesion (right column). The numbers at left represent the distance in millimeters from the midline. Roman numerals represent lobule designations according to Larsell (1970). ICP, $M C P$, and $S C P$ represent inferior, middle, and superior cerebellar peduncles, respectively.

tex is necessary for the induction of plasticity that mediates short-latency responses.

These findings are consistent with previous observations regarding cerebellar cortex and adaptation of the vestibulo-ocular reflex (Raymond and Lisberger, 1996). Cerebellar cortex lesions have been shown to affect only partially the expression of previously learned VOR gain adaptation but to prevent subsequent VOR adaptation (Robinson, 1976). The present data together with the VOR studies suggest a consistent picture in which cerebellar cortex is required for learned increases and decreases (acquisition and extinction) in cerebellar-dependent movements.

Despite this concordance, many previous eyelid conditioning studies contradict this view. McCormick and Thompson (1984a) reported that cerebellar cortex lesions do not affect conditioned response expression. Subsequent studies have reported that cerebellar cortex lesions do not affect expression or extinction and have only marginal effects on the acquisition of responses (McCormick and Thompson, 1984a; Woodruff-Pak et al., 1985, 1993; 


\section{PRE LESION CS}
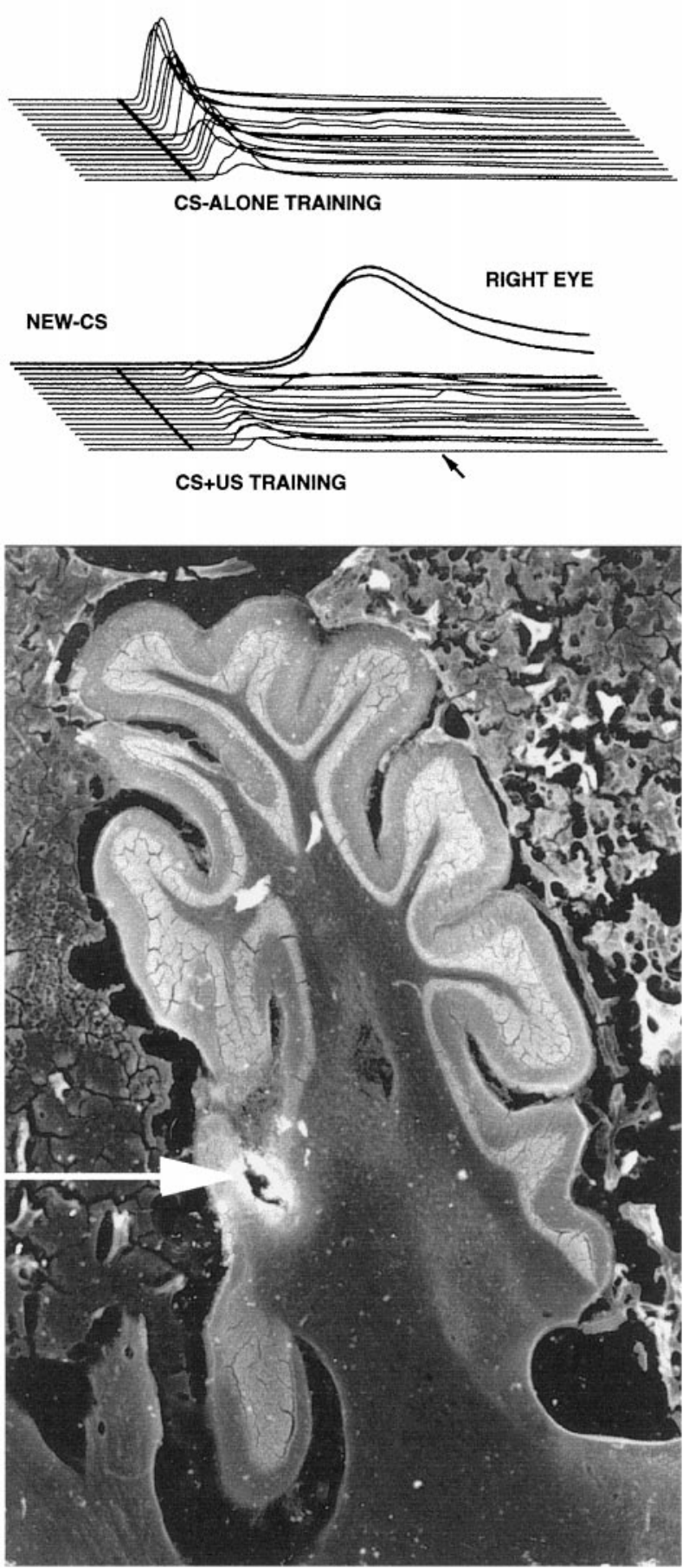

Figure 5. Data from the animal with the smallest effective lesion. Bottom, A parasagital section through the region with the electrolytic lesion is shown. Top, Middle, Each sweep shown is the average of the CS-alone probe trials from each daily session. Top, These sweeps show the shortlatency responses elicited by the original CS. If anything, these responses increased over the 15 sessions, during which the CS was never paired with the US. Middle, These sweeps show the responding to the new CS. There are small short-latency responses; presumably these result from stimulus generalization. However, neither the amplitude nor frequency of these responses increases over the $15 \mathrm{~d}$ of training. In contrast, the last two sweeps (RIGHT EYE) show the rapid acquisition of properly timed responses when training was switched to the contralateral eye.
Lavond et al., 1987; Knowlton et al., 1988; Lavond and Steinmetz, 1989; Yeo and Hardiman, 1992; Harvey et al., 1993; Clark and Lavond, 1994; Logan et al., 1994). However, pharmacological block of cerebellar cortex output, which abolished conditioned response timing, indicates the effects of cerebellar cortex lesions independent of any knowledge of which regions of cortex are important (Garcia and Mauk, 1998b). Thus, a lesion in the region of cerebellar cortex involved in eyelid conditioning should have the same result. We have shown here and previously that lesions of the anterior lobe produce this effect on response timing (Perrett et al., 1993; Perrett and Mauk, 1995). Thus, previous findings that cerebellar cortex lesions did not affect response timing, acquisition, or extinction appear to have been false negatives. Indeed, the present results replicated these negative findings when the lesions did not affect response timing. Similarly, our data suggest that the abolition of conditioned responses by cerebellar cortex lesions reported in other previous studies (Yeo et al., 1984, 1985b; Yeo and Hardiman, 1992) must have been the result of unintended damage to underlying pathways necessary for response expression. Here, by using the status of previously trained responses as an index for the functional extent and specificity of a cerebellar cortex lesion, we can exclude confounds that may have led previously to false positives or negatives.

As with all lesions caution is required regarding secondary effects of these lesions. One such concern relates to the possible contributions of degeneration of CS and US pathways produced by the lesions. Here, degeneration of CS pathways can be excluded by the presence of conditioned responses elicited by the previously trained CS. Degeneration of the US pathway, the climbing fibers, cannot be so easily excluded. It remains possible therefore that the cerebellar nucleus may be capable of learning without the cerebellar cortex. The absence of learning with cerebellar cortex lesions could then result from degeneration of climbing fiber inputs to the nucleus. This issue may require reversible lesion studies to resolve. Another potential concern is abnormal activity of nucleus cells after the lesions, which might prevent learning. However, such activity should be apparent behaviorally, because activation of nucleus cells can elicit eyelid responses in untrained animals. In ongoing reversible lesion studies we sometimes observe tonic partial closure of the eyelid, possibly because of high levels of activity in nucleus cells (unpublished data). No such tonic eyelid closure was observed in the present studies, consistent with an absence of abnormal nucleus cell activity after the lesions.

Our data also seemingly contradict results from eyelid conditioning in mutant mice in which the Purkinje cells undergo degeneration. These Purkinje cell degeneration mice show poor, but not abolished, acquisition at a time when Purkinje cells are almost completely gone (Chen et al., 1996, 1999). Our results provide no explanation for these differences. It is possible that there are enough viable Purkinje cells remaining in these mice to support compromised learning, that there are species differences, or that the mutations cause compensatory changes that allow the cerebellar nuclei or other brain regions to operate abnormally (Steele et al., 1998). Although the present results do not support or deny these possibilities, they do provide assurances that the lesions damaged the anterior lobe enough to affect response timing without significant damage to pathways required for response expression. 


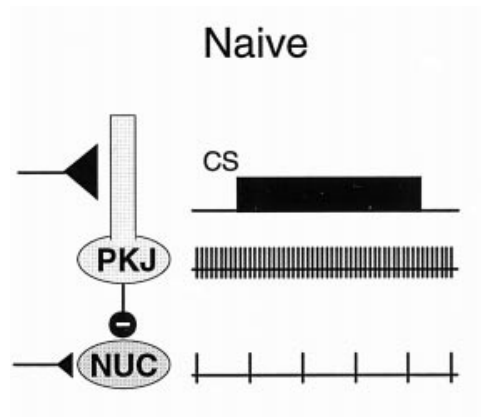

Response
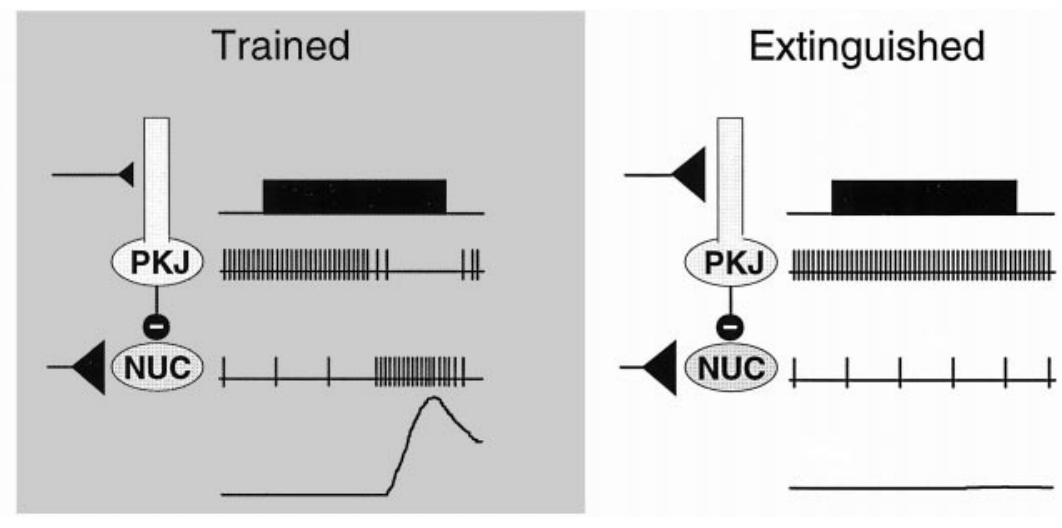

Figure 6. A schematic representation of the event hypothesized to mediate the acquisition and extinction of conditioned eyelid responses. The key elements are the two modifiable synapse types shown as dark triangles: plasticity is assumed to occur at the CS-activated synapses between granule cells and Purkinje cells in the cerebellar cortex and at CS-activated synapses between mossy fibers and nucleus cells. In both cases the relative strength of the synapse is illustrated by the size of the triangle; e.g., larger is stronger. Left, Initially, the CS-activated granule cell synapses are relatively strong, and the CS-activated mossy fiber synapses are relatively weak. Presentation of the CS would have little effect on nucleus cell activity; the weak excitation from mossy fibers would be counteracted by the ongoing activity of Purkinje cells, which is not changed significantly by the CS. Middle, After training, the granule cell synapses are weaker because of LTD, and the mossy fiber synapses are stronger because of LTP. Now, presentation of the CS can produce robust activity in the nucleus cell. Right, In extinction, the granule cell synapses have become stronger because of LTP. These hypothesized events are consistent with the dependence of acquisition and extinction on the cerebellar cortex. NUC, Nucleus; PKJ, Purkinje cell.

Combined with previous studies the present results support the assertions that (1) the cerebellar cortex is not necessary for the expression of previously learned conditioned responses if timing is ignored but that (2) the cerebellar cortex is necessary for the acquisition and extinction of conditioned eyelid responses. Although the effect of cerebellar cortex lesions on the timing of VOR has not been tested, these assertions are otherwise consistent with the effects of cerebellar cortex lesions on adaptation of the VOR. These assertions are also consistent with the contributions to eyelid responses suggested by stimulation and recording studies. Purkinje cell recordings from the region of ferret cerebellar cortex involved in eyelid responses display learned decreases in Purkinje cell activity during the expression of conditioned responses (Hesslow and Ivarsson, 1994). Moreover, microstimulation of these regions suppresses the expression of conditioned responses (Hesslow, 1994). These results indicate that Purkinje cell activity suppresses the ability to express eyelid responses and that the expression of conditioned responses requires the acquisition of decreases in Purkinje cell activity in response to the CS. Our results suggest that in the absence of these processes, plasticity in other regions either does not occur or is incapable of supporting expression of conditioned responses.

The effects of cerebellar cortex lesions on response timing, acquisition, and extinction have important implications about cerebellar sites and rules for plasticity. Because conditioned response timing is learned, the effects of cerebellar cortex lesions imply that there are at least two cerebellar sites of plasticity responsible for eyelid conditioning. The abolition of timing implies timing-related plasticity either in the cerebellar cortex itself or at sites afferent to the cerebellum. In the latter view timing information would be conveyed to the cerebellar cortex via timed (delayed) activation of mossy fiber inputs by the CS. However, because mossy fiber inputs also project to the interpositus nucleus, this view predicts that responses should be appropriately delayed despite removal of cerebellar cortex. The data therefore appear to support more strongly the view that the cerebellar cortex is the site at which temporal coding and timing-related plasticity occurs. Similarly, the presence of (improperly timed) responses after cerebellar cortex removal indicates learningrelated plasticity in the cerebellar nucleus as well. Moreover, the inability to acquire responses without the cerebellar cortex suggests that the induction of plasticity in the nucleus requires intact input from Purkinje cells, as predicted by Miles and Lisberger (1981).

On the basis of these arguments, we have proposed a model to account for the acquisition, properly timed expression, and extinction of conditioned eyelid responses (Mauk and Donegan, 1997; Mauk et al., 1997). As shown schematically in Figure 6, the initial lack of responding to the CS may be caused by two factors: the relatively weak excitatory connections between CS-activated mossy fibers and the nucleus cells and the relatively strong connections between the CS-activated granule cells and the Purkinje cells. When the CS is presented, the Purkinje cells maintain their strong ongoing activity owing to the relatively strong granule cell synapses. The corresponding inhibition of the nucleus cells would counteract any weak excitation from the CS-activated mossy fiber inputs. With repeated CS + US pairings, the CS-activated granule cell synapses would undergo long-term depression (LTD), leading to a learned pause in Purkinje activity during the CS. The appropriate timing of this pause might be due to different granule cells becoming active at different times during the CS (Buonomano and Mauk, 1994; Raymond et al., 1996; Mauk, 1997; Mauk et al., 1998).

We suggest that this learned pause in Purkinje cell activity during the CS may serve two purposes. First, it would disinhibit nucleus cells, making it possible to elicit a response. Second, it may signal the induction of a long-term potentiation (LTP)-like phenomenon at CS-activated mossy fiber synapses in the nucleus. As such, presentation of the CS to well trained animals would provide a strong excitatory input to the nucleus directly from the strengthened mossy fiber synapses and would provide a well timed pause in inhibition because of the plasticity in the cerebellar cortex. This hypothesis is consistent with a number of findings. It would explain the well timed responses seen in intact animals, the short-latency responses after cerebellar cortex lesions, and the inability to acquire new responses without the cerebellar cortex. 
We have further hypothesized that extinction may result from the induction of LTP at the synapses between CS-activated granule cells and Purkinje cells. This would restore the ongoing activity of the Purkinje cells during the CS, and the corresponding inhibition of nucleus cells would prevent responding. This hypothesis is consistent with the requirement of the cerebellar cortex for the extinction of conditioned responses (Perrett and Mauk, 1995).

Confidence in these hypotheses clearly requires further empirical test. For now, however, they are consistent with the general notion that changes in cerebellar-mediated responses, such as increasing or decreasing the gain of the VOR as well as acquisition or extinction of conditioned eyelid responses, require an intact cerebellar cortex.

\section{REFERENCES}

Buonomano DV, Mauk MD (1994) Neural network model of the cerebellum: temporal discrimination and the timing of motor responses. Neural Comput 6:38-55.

Chapman PF, Steinmetz JE, Thompson RF (1988) Classical conditioning does not occur when direct stimulation of the red nucleus or cerebellar nuclei is the unconditioned stimulus. Brain Res 442:97-104.

Chapman PF, Steinmetz JE, Sears LL, Thompson RF (1990) Effects of lidocaine injection in the interpositus nucleus and red nucleus on conditioned behavioral and neuronal responses. Brain Res 537:149-156.

Chen L, Bao S, Thompson RF (1999) Bilateral lesions of the interpositus nucleus completely prevent eyeblink conditioning in Purkinje celldegeneration mutant mice. Behav Neurosci 113:204-210.

Chen WR, Lee S, Kato K, Spencer DD, Shepherd GM, Williamson A (1996) Long-term modifications of synaptic efficacy in the human inferior and middle temporal cortex. Proc Natl Acad Sci USA 93:8011-8015.

Clark RE, Lavond DG (1994) Reacquisition of eyeblink classical conditioning following large cerebellar cortical lesions in Dutch belted rabbits. Behav Brain Res 61:101-106.

Garcia KS, Mauk MD (1998a) Cerebellar cortex is necessary for acquisition of Pavlovian eyelid responses. Soc Neurosci Abstr 24:444.

Garcia KS, Mauk MD (1998b) Pharmacological analysis of cerebellar contributions to the timing and expression of conditioned responses. Neuropharmacology 37:471-480.

Gilbert PF, Thach WT (1977) Purkinje cell activity during motor learning. Brain Res 128:309-328.

Gruart A, Yeo CH (1995) Cerebellar cortex and eyeblink conditioning: bilateral regulation of conditioned responses. Exp Brain Res 104:431-448.

Harvey JA, Welsh JP, Yeo CH, Romano AG (1993) Recoverable and nonrecoverable deficits in conditioned responses after cerebellar cortical lesions. J Neurosci 13:1624-1635.

Hesslow G (1994) Inhibition of classically conditioned eyeblink responses by stimulation of the cerebellar cortex in the decerebrate cat. J Physiol (Lond) 476:245-256.

Hesslow G, Ivarsson M (1994) Suppression of cerebellar Purkinje cells during conditioned responses in ferrets. NeuroReport 5:649-652.

Ito M (1993) Cerebellar flocculus hypothesis. Nature 363:24-25.

Knowlton BJ, Lavond DG, Thompson RF (1988) The effect of lesions of cerebellar cortex on retention of the classically conditioned eyeblink response when stimulation of the lateral reticular nucleus is used as the conditioned stimulus. Behav Neural Biol 49:293-301.

Krupa DJ, Thompson JK, Thompson RF (1993) Localization of a memory trace in the mammalian brain. Science 260:989-991.

Larsell 0 (1970) The comparative anatomy and histology of the cerebellum from monotremes through apes. Minneapolis: University of Minnesota.

Lavond DG, Steinmetz JE (1989) Acquisition of classical conditioning without cerebellar cortex. Behav Brain Res 33:113-164.

Lavond DG, Steinmetz JE, Yokaitis MH, Thompson RF (1987) Reacquisition of classical conditioning after removal of cerebellar cortex. Exp Brain Res 67:569-593.

Lisberger SG (1988) The neural basis for learning of simple motor skills. Science 242:728-735.
Lisberger SG, Raymond JL (1996) Neural recordings and behavioral observations in the monkey vestibulo-ocular reflex constrain the cellular mechanisms for cerebellum-dependent behavioral learning. J Physiol (Paris) 90:381-382.

Lisberger SG, Sejnowski TJ (1992) Motor learning in a recurrent network model based on the vestibulo-ocular reflex. Nature 360:159-161.

Logan CG, Lavond DG, Wong JT, Thompson RF (1994) Acquisition of classically conditioned eyeblink response following bilateral lesions of flocculus and paraflocculus. Behav Neural Biol 61:102-106.

Marr D (1969) A theory of cerebellar cortex. J Physiol (Lond) 202:437-470.

Mauk MD (1997) Roles of cerebellar cortex and nuclei in motor learning: contradictions or clues? Neuron 18:343-346.

Mauk MD, Donegan NH (1997) A model of Pavlovian eyelid conditioning based on the synaptic organization of the cerebellum. Learn Mem 3:130-158.

Mauk MD, Steinmetz JE, Thompson RF (1986) Classical conditioning using stimulation of the inferior olive as the unconditioned stimulus. Proc Natl Acad Sci USA 83:5349-5353.

Mauk MD, Steele PM, Medina JF (1997) Cerebellar involvement in motor learning. The Neuroscientist 3:303-313.

Mauk MD, Garcia KS, Medina JF, Steele PM (1998) Does cerebellar LTD mediate motor learning? Toward a resolution without a smoking gun. Neuron 20:359-362.

McCormick DA, Thompson RF (1984a) Cerebellum: essential involvement in the classically conditioned eyelid response. Science 223:296-299.

McCormick DA, Thompson RF (1984b) Neuronal responses of the rabbit cerebellum during acquisition and performance of a classically conditioned nictitating membrane-eyelid response. J Neurosci $4: 2811-2822$.

McCormick DA, Steinmetz JE, Thompson RF (1985) Lesions of the inferior olivary complex cause extinction of the classically conditioned eyeblink response. Brain Res 359:120-130.

Miles FA, Lisberger SG (1981) Plasticity in the vestibulo-ocular reflex: a new hypothesis. Annu Rev Neurosci 4:273-299.

Perrett SP, Mauk MD (1995) Extinction of conditioned eyelid responses requires the anterior lobe of cerebellar cortex. J Neurosci 15:2074-2080.

Perrett SP, Ruiz BP, Mauk MD (1993) Cerebellar cortex lesions disrupt learning-dependent timing of conditioned eyelid responses. J Neurosci 13:1708-1718.

Raymond JL, Lisberger SG (1996) Behavioral analysis of signals that guide learned changes in the amplitude and dynamics of the vestibuloocular reflex. J Neurosci 16:7791-7802.

Raymond JL, Lisberger SG, Mauk MD (1996) The cerebellum: a neuronal learning machine? Science 272:1126-1131.

Ritchie L (1976) Effects of cerebellar lesions on saccadic eye movements. J Neurophysiol 39:1246-1256.

Robinson DA (1976) Adaptive gain control of vestibuloocular reflex by the cerebellum. J Neurophysiol 39:954-969.

Robinson FR, Straube A, Fuchs AF (1993) Role of the caudal fastigial nucleus in saccade generation. II. Effects of muscimol inactivation. J Neurophysiol 70:1741-1758.

Steele PM, Medina JF, Nores WL, Mauk MD (1998) Using genetic mutations to study the neural basis of behavior. Cell 95:879-882.

Steinmetz JE, Rosen DJ, Chapman PF, Lavond DG, Thompson RF (1986) Classical conditioning of the rabbit eyelid response with a mossy-fiber stimulation CS. I. Pontine nuclei and middle cerebellar peduncle stimulation. Behav Neurosci 100:878-887.

Steinmetz JE, Logan CG, Rosen DJ, Thompson JK, Lavond DG, Thompson RF (1987) Initial localization of the acoustic conditioned stimulus projection system to the cerebellum essential for classical eyelid conditioning. Proc Natl Acad Sci USA 84:3531-3535.

Steinmetz JE, Lavond DG, Thompson RF (1989) Classical conditioning in rabbits using pontine nucleus stimulation as a conditioned stimulus and inferior olive stimulation as an unconditioned stimulus. Synapse $3: 225-233$

Thach WT (1980) Complex spikes, the inferior olive, and natural behavior. In: The inferior olivary nucleus: anatomy and physiology (Courville J, de Montigny C, Lamarre Y, eds), pp 349-360. New York: Raven.

Thompson RF (1986) The neurobiology of learning and memory. Science 233:941-947.

Thompson RF, Krupa DJ (1994) Organization of memory traces in the mammalian brain. Annu Rev Neurosci 17:519-549. 
Tracy JA, Thompson JK, Krupa DJ, Thompson RF (1998) Evidence of plasticity in the pontocerebellar conditioned stimulus pathway during classical conditioning of the eyeblink response in the rabbit. Behav Neurosci 112:267-285.

Woodruff-Pak DS, Lavond DG, Thompson RF (1985) Trace conditioning: abolished by cerebellar nuclear lesions but not lateral cerebellar cortex aspirations. Brain Res 348:249-260.

Woodruff-Pak DS, Lavond DG, Logan CG, Steinmetz JE, Thompson RF (1993) Cerebellar cortical lesions and reacquisition in classical conditioning of the nictitating membrane response in rabbits. Brain Res 608:67-77.

Yeo CH, Hardiman MJ (1992) Cerebellar cortex and eyeblink conditioning: a reexamination. Exp Brain Res 88:623-638.
Yeo CH, Hardiman MJ, Glickstein M (1984) Discrete lesions of the cerebellar cortex abolish the classically conditioned nictitating membrane response of the rabbit. Behav Brain Res 13:261-266.

Yeo CH, Hardiman MJ, Glickstein M (1985a) Classical conditioning of the nictitating membrane response of the rabbit. I. Lesions of the cerebellar nuclei. Exp Brain Res 60:87-98.

Yeo CH, Hardiman MJ, Glickstein M (1985b) Classical conditioning of the nictitating membrane response of the rabbit. II. Lesions of the cerebellar cortex. Exp Brain Res 60:99-113.

Yeo CH, Hardiman MJ, Glickstein M (1986) Classical conditioning of the nictitating membrane response of the rabbit. IV. Lesions of the inferior olive. Exp Brain Res 63:81-92. 